\title{
“虚拟教室与教学楼” 模型在无机化学四个量子数教学中的应用
}

陈广慧 ${ }^{1}$, 林旺强 ${ }^{1}$, 马荔 $2,{ }^{\star}$

1 汕头大学理学院化学系, 广东汕头 515063

2 上海交通大学化学化工学院, 上海 200240

摘要: 经过多年无机化学课程的教学, 发现很多学生对物质结构内容中有关四个量子数部分一知半解, 严重影响后续 内容的学习。为了帮助学生走出困境, 我们采用类比教学方法利用 “虚拟教室与教学楼” 模型将抽象的四个量子数的 内容具象化。结果发现学生的掌握程度比往年的都要理想, 说明当前类比教学法取得了满意的教学效果。

关键词: 虚拟教室与教学楼; 无机化学; 四个量子数; 类比教学方法

中图分类号: G64; O6

\section{Application of the "Virtual Classroom and Teaching Building" Model to the Teaching of the Four Quantum Numbers in Inorganic Chemistry}

\author{
Guanghui Chen ${ }^{1}$, Wangqiang Lin ${ }^{1}$, Li Ma ${ }^{2, *}$ \\ ${ }^{1}$ Department of Chemistry, College of Science, Shantou University, Shantou 515063, Guangdong Province, China. \\ ${ }^{2}$ School of Chemistry and Chemical Engineering, Shanghai Jiao Tong University, Shanghai 200240, China.
}

\begin{abstract}
After years of teaching inorganic chemistry, it is found that many students lack knowledge of the four quantum numbers in the content of material structure, which seriously affects their learning of the following content. In order to help students to get out of the dilemma, we use analogy teaching method and use the "virtual classroom and teaching building" model to visualize the content of the abstract four quantum numbers. The results show that the students' mastery degree is better than that of previous years, which indicates that the current analogy teaching method has achieved satisfactory teaching effect.
\end{abstract}

Key Words: Virtual classroom and teaching building; Inorganic chemistry; Four quantum numbers;

Method of analogy teaching

\section{1 课程调研}

无机化学 ${ }^{[1]}$ 是化学专业大一本科生的必修课。根据多年的教学经验, 发现其中许多学生对主量 子数 $n$ 、角量子数 $l$ 、磁量子数 $m$ 和自旋磁量子数 $m_{s}$ 这四个量子数的概念及其物理意义不理解, 影响了 后续内容, 如亚层原子轨道的能量比较、核外电子的排布、元素各论知识的学习。为了解学生陷入 困境的原因, 我们进行了课程调研, 探究是什么原因导致对以上内容理解困难。最后, 根据学生的 反馈，总结出以下两点原因:

(1) 大部分学生在高中阶段原子结构与性质相关知识的缺失。广东高校的大部分生源均来自于

收稿: 2020-08-07; 录用: 2020-10-19; 网络发表: 2020-12-10

*通讯作者, Email: mali@sjtu.edu.cn

基金资助: 2018 年广东省高等教育教学改革项目 
广东省, 高考使用的是全国I卷, 其中原子结构与性质的内容不是必考, 只需从两道选考题, 即有机 化学基础和原子结构与性质中任选一道答题即可。因为有机化学基础选做题相对比较简单, 相对容 易得分, 所以广东省内的大部分高中都建议或引导学生专攻有机化学基础的选做题, 甚至不少高中 直接放弃原子结构与性质的内容。只有少数重点高中为了取得优异的竞赛成绩, 针对有机化学基础 和原子结构与性质的内容安排课时进行学习。因此, 导致大部分高中学生对于原子结构的知识还停 留在初中阶段, 认为核外电子只是单纯地绕原子核做圆周运动, 完全没有掌握亚层轨道、电子自旋 这些基本概念。由于高中原子结构与性质相关知识的缺失, 这部分学生初到大学, 以前学习的知识 体系突然被颠覆, 面对无机化学课程, 特别是原子结构与性质的内容就会束手无策, 觉得难懂、难 学。

(2) 学生还没适应大学学习的抽象思维。高中的大部分知识都非常具体, 着重学习现象与结论, 例如 $\mathrm{A}$ 和 $\mathrm{B}$ 反应生成 $\mathrm{C}$ 和 $\mathrm{D}$ 并伴随着怎样的实验现象, 对于反应机理或物质内在结构的学习只是停留 在皮毛阶段。然而大学着重学习原理和结构与性质内在的联系, 需要具备抽象的思维来理解原理。 而大一学生刚从高中毕业, 还没有具备大学化学专业课学习所必需的抽象思维能力。

根据以上调研可以发现, 无机化学课程是化学类专业大一新生踏入大学的第一门专业课, 教师 在教学中需要深入了解学生现有的知识水平, 对接高中的知识, 尽量想办法把抽象的概念具象化, 并在教学中逐渐培养学生的抽象思维, 这是对无机化学课程教师的一大挑战。

研究表明 ${ }^{[2]}$, 学生在学习的过程中看到陌生概念和规律时, 总是不断在大脑中搜索与此相关的 信息, 再根据相关信息进行篮选和处理。当发现新问题与旧知识有相似点时, 会进行比较和推测, 使用类比法正是遵循这个过程。类比的一个重要特征就是用一个熟悉的事物去说明陌生的事物, 用浅显的事理来说明复杂抽象的事理, 达到其他逻辑推理方法所不能达到的目的。因此, 使用类比 法不仅遵循学生的学习规律, 同时也能锻炼学生的创造性思维能力。所以, 类比教学对教育的改革 和发展有着重要的实践意义。

采用类比法教学使课程中抽象的概念具象化, 很多高校化学教师积累了成功的经验。例如郭玉 䴕[3]使用类比法应用在物理化学热力学函数关系式的教学中, 帮助学生记忆复杂的热力学函数关系 式, 最终帮助学生提高学习效果。杨风霞和连照勋 ${ }^{[4]}$ 采用公式类比、模型类比、概念类比和判据类比 的方法对物理化学知识进行总结与归纳, 便于学生掌握和复习。黄四平等 ${ }^{[5]}$ 将类比思维应用于有机 化学教学中, 有效地提高教学效果和学生的学习效果, 拓宽学生的知识视野、培养学生的创新思维。 唐正姣等 ${ }^{[6]}$ 将类比教学应用于化工原理课程中, 引导学生使用类比法解决填料吸收塔与板式精馏塔 的计算问题, 达到提高教学效果的目的。借鉴他们的成功经验, 我们也尝试将类比法应用于无机化 学物质结构内容的教学中, 将抽象的概念具体化、形象化, 希望通过类比教学能使学生对四个量子 数的概念有深入的理解和掌握。

\section{2 四个量子数的物理意义}

进行类比法教学前, 需要理解四个量子数的由来: 推导和求解单电子原子的薛定谔方程 (Schrödinger equation) ${ }^{[7], ~}$ 如式(1)所示:

$$
\hat{H} \psi=E \psi
$$

式中 $\hat{H}$ 为总能量Hamilton算符, $\psi$ 为单电子原子波函数, $E$ 为单电子原子能量的本征值。其中, 总能 量Hamilton算符 $\hat{H}$ 的表达式 ${ }^{[8]}$ 如式(2)所示:

$$
\hat{H}=\hat{T}+\hat{V}=-\frac{h^{2}}{8 \pi^{2} \mu}\left(\frac{\partial^{2}}{\partial x^{2}}+\frac{\partial^{2}}{\partial y^{2}}+\frac{\partial^{2}}{\partial z^{2}}\right)-\frac{Z e^{2}}{4 \pi \varepsilon_{0} r}
$$

式中 $\hat{T}$ 为动能算符; $\hat{V}$ 为势能算符; $h$ 为普朗克常数, 其值为 $6.6262 \times 10^{-34} \mathrm{~J} \cdot \mathrm{s} ; \pi$ 为圆周率; $x 、 y$ 和 $z$ 为三维立体坐标轴; $\varepsilon_{0}$ 为真空介电常数, 其值为 $8.854 \times 10^{-12} \mathrm{~J}^{-1} \cdot \mathrm{C}^{2} \cdot \mathrm{m}^{-1}, Z$ 为核电荷数; $r$ 为电子到 核的距离。 $\mu$ 为原子的折合质量 ${ }^{[9]}$, 如式(3)所示: 


$$
\mu=\frac{m_{\mathrm{e}} m_{\mathrm{N}}}{m_{\mathrm{e}}+m_{\mathrm{N}}}
$$

式中 $m_{\mathrm{e}}$ 与 $m_{\mathrm{N}}$ 分别为电子和原子核的质量。因此, 将式(2)代入式(1)即可得到单电子原子的薛定谔方 程, 如式(4)所示:

$$
\left[-\frac{h^{2}}{8 \pi^{2} \mu} \nabla^{2}-\frac{Z e^{2}}{4 \pi \varepsilon_{0} r}\right] \psi=E \psi
$$

其中, $\nabla^{2}=\left(\frac{\partial^{2}}{\partial x^{2}}+\frac{\partial^{2}}{\partial y^{2}}+\frac{\partial^{2}}{\partial z^{2}}\right)$, 为Laplace算符[10], 将如式(4)所示的单电子原子薛定谔方程求解即 可得到主量子数 $n$ 、角量子数 $l$ 和磁量子数 $m$ 。

实验证明, 使用分辨率较强的分光镜观察氢原子光谱时, 当电子由 $2 p$ 轨道跃迁到 $1 s$ 轨道时得到的 不是一条谱线, 而是靠得很近的两条谱线。这一现象不能用 $n, l, m$ 三个量子数进行解释。因为 $2 p$ 和 $1 s$ 从 $n, l, m$ 三个量子数的角度来看都只是一个能级, 这种跃迁只能产生一条谱线。1925年两位荷兰 学生乌伦贝克(George Eugene Uhlenbeck)和古德斯密特(Samuel Abraham Goudsmit)根据氢原子和碱金 属光谱的精细结构等诸多实验事实 ${ }^{[11]}$, 发展了行星模型, 提出电子不仅有轨道运动、还有自旋运动具 有自旋角动量这一假说。自旋角动量在磁场方向的分量 $M_{s z}$ 由自旋磁量子数 $m_{s}$ 决定 ${ }^{[12]}$, 如式(5)所示:

$$
M_{s z}=m_{s} \frac{h}{2 \pi}
$$

式(5)中自旋磁量子数 $m_{s}$ 只有两个数值, 即 $m_{s}= \pm \frac{1}{2}$ 。因此电子的自旋方式只有两种, 分别是自旋向 上和自旋向下。因此正是电子具有两种不同的自旋方式，才导致了氢原子光谱的精细结构。

根据以上单电子原子薛定谔方程的求解即可得到四个量子数的物理意义。主量子数 $n$ 是描述原 子中电子出现概率最大区域离核的远近 ${ }^{[1]} 。 n=1$, 代表第一层, 这是离核最近的电子层; $n=2$, 代 表第二层; $n=3$, 代表第三层。因此, $n$ 值是量子化的, $n$ 值越大代表电子离核越远, 能量越高。对 于单电子的氢原子和类氢离子来说, 主量子数 $n$ 决定了电子的能量; 对于多电子原子或离子, 核外电 子的能量除了取决于主量子数 $n$ 以外, 还与其他因素有关, 如角量子数 $l$ 。

角量子数 $l$ 的取值为 $0,1,2,3,4, \cdots,(n-1)$, 分别用符号 $s, p, d, f, g$ 来表示。因此, $l$ 的 取值受主量子数 $n$ 的限制, 只能取从 0 到 $(n-1)$ 的整数, 共有 $n$ 个值。角量子数 $l$ 的物理意义是代表原子 亚层轨道的形状。 $l=0$, 表示 $s$ 轨道, 形状为球形; $l=1$, 表示 $p$ 轨道, 形状为哑铃形; $l=2$, 表示 $d$ 轨道, 形状为花瓣形; $l=3$, 表示 $f$ 轨道, 形状更复杂。对于多电子原子或离子来说, 其能量由主量 子数 $n$ 和角量子数 $l$ 共同决定 ${ }^{[13]}$ 。对于 $n$ 相同， $l$ 不同的原子轨道， $l$ 越大轨道能量 $E$ 越大，例如 $E_{4 s}<E_{4 p}<$ $E_{4 d}<E_{4 f}$ 。但是对于单电子原子或离子, 如氢原子, 其能量 $E$ 不受 $l$ 的影响, 只跟 $n$ 有关, 即 $E_{n s}=E_{n p}=$ $E_{n d}=E_{n f}{ }^{[1]}$ 。

磁量子数 $m$ 的取值为 $0, \pm 1, \pm 2, \pm 3, \pm 4, \cdots, \pm l$, 即 $m$ 的取值受角量子数 $l$ 的影响, 从 0 到 $\pm l$, 共 有 $(2 l+1)$ 个取值 ${ }^{[14]}$ 。磁量子数 $m$ 的物理意义是决定原子轨道在核外空间中的取向 ${ }^{[8]}$ 。当角量子数 $l=$ 0 时, 表示球形的 $s$ 轨道, 这时磁量子数 $m$ 只有一种取值 0 , 因此 $s$ 轨道在核外空间中只有一种分布方向, 即以核为球心的球形分布; $l=1$ 时, 表示哑铃形的 $p$ 轨道, $m$ 有三种取值 0 和 \pm 1 , 说明 $p$ 轨道在核外空 间坐标系有三种不同的分布方向。一般情况下, 磁量子数 $m$ 与原子轨道的能量无关, 因此三种不同取 向的 $p$ 轨道, 其能量相等; $l=2$ 时, 表示花瓣形的 $d$ 轨道, $m$ 有五种取值 $0, \pm 1$ 和 \pm 2 , 说明在核外空间 中有五种不同的分布方向, 这 5 种 $d$ 轨道能量简并, 即简并度为 5 。

自旋磁量子数 $m_{s}$ 的取值只有两个, 即 $\pm \frac{1}{2}$, 表示的是电子的自旋方向。若电子是自旋向上, 则 $m_{s}=$ $+\frac{1}{2}$; 若电子是自旋向下, 则 $m_{s}=-\frac{1}{2}$ 。根据泡利不相容原理, 同一原子轨道上最多只能容纳自旋方 式不同的 2 个电子 ${ }^{[15]}$, 例如 $l=0$ 时, $m=0$, 只有一个原子轨道, 因此 $s$ 轨道最多只能容纳两个电子, 
分别是自旋向上和自旋向下; $l=2$ 时, $m$ 取值为 $0, \pm 1$ 和 \pm 2 , 一共有 5 个原子轨道, 因此 $d$ 轨道最多只 能容纳 10 个电子。

综上所述, $n, l, m$ 这三个量子数可以决定原子轨道的离核远近、形状和空间取向, 而 $m_{s}$ 则决定 电子的自旋方式。因此, 四个量子数确定之后, 电子在核外空间的运动状态就确定了。

\section{3 四个量子数的类比教学}

如果将以上抽象的知识直接向刚进大学的大一新生进行讲授, 学生一时肯定难以接受新概念和 新知识, 例如原子轨道代表原子体系单电子波函数等概念。因此, 为了帮助学生越过抽象思维的 “能 垒”, 我们先采用类比教学的方法向学生构建 “虚拟教室与教学楼” 模型将四个量子数的概念及其物 理意义具象化。

对于单原子或离子, 如氢原子或者类氢离子, 无论主量子数 $n$ 等于多少, 原子亚层轨道的能量都 相同, 即 $E_{n s}=E_{n p}=E_{n d}=E_{n f}$ 。因此, 我们向学生构建出 “虚拟教室与教学楼” 模型, 如图 1所示, 用 来说明四个量子数的概念。将电子比作学生, 楼层比作电子层, 楼层中的复式比作电子亚层, 一间 教室比作一个原子轨道。当 $n=1$ 时, 只有 1 个 $s$ 轨道, 对应教学楼一楼只有一间教室, 教室里面有两 位学生, 一位学生面向黑板, 另一位学生背向黑板, 代表 $1 s$ 轨道有两个自旋相反的电子; $n=2$ 时, 对应教学楼二楼有四间教室, 分别是一间 $2 s$ 教室和三间 $2 p$ 教室, 每间教室有两个坐向相反的座位; 同理, $n=3$ 时, 教学楼三楼有一间 $3 s$ 教室、三间 $3 p$ 教室和五间 $3 d$ 教室, 每间教室有两个坐向相反的 座位。 $n \geq 4$ 的情况也是以此类推。

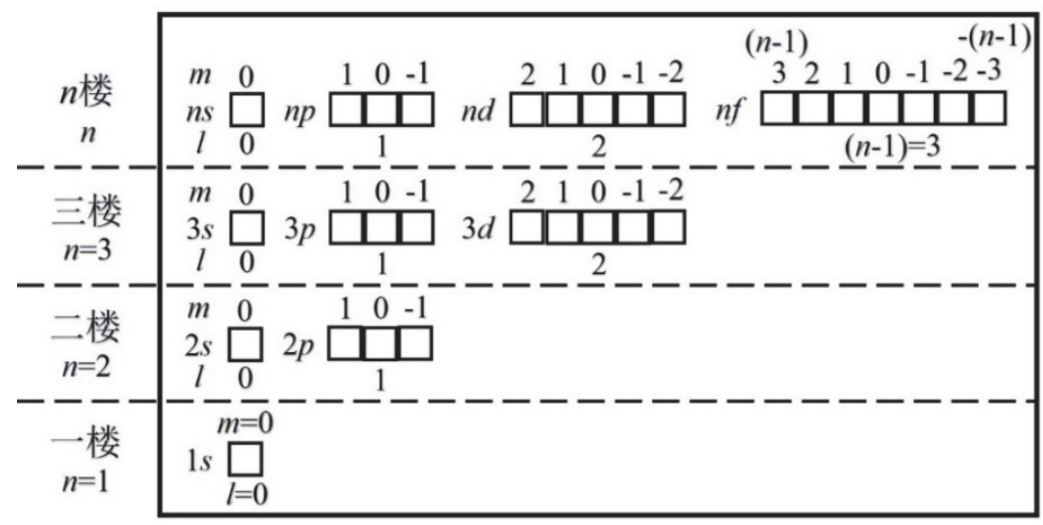

图1 单电子体系 “虚拟教室与教学楼” 模型

对于多电子原子或离子, 结合图 2 “虚拟教室与教学楼” 模型进行类比教学。 $n=1$ 时, 只有 1 个 $s$ 轨道, 对应教学楼一楼只有一间教室, 教室里面有两位坐向相反学生, 代表 $1 s$ 轨道填充两个自旋相 反的电子; $n=2$ 时, 有 1 个 $2 s$ 轨道和 3 个 $2 p$ 轨道, 对于多电子原子或离子 $2 p$ 轨道的能量大于 $2 s$ 轨道, 因 此我们将复式楼层比喻为电子亚层: 教学楼二楼是二层复式楼层, 复式一层只有一间教室, 代表 $2 s$ 轨道, 教室里有两个坐向相反的座位, 代表可容纳两个自旋相反的电子; 复式二层有三间教室, 代 表 $2 p$ 三个能量简并的轨道, 每间教室里面有两个坐向相反的座位, 复式二层共4间教室, 教室数等于 $n^{2}(n=2)$; 同理, $n=3$ 时, 教学楼三楼是三层复式楼层, 复式一层只有一间教室, 二层有三间教室, 三层有五间教室，复式二层共 9 间教室，教室数等于 $n^{2}(n=3) ; n \geq 4$ 的情况也是依此类推。

与此同时, 我们在讲授时特别强调主量子数、角量子数与磁量子数间的约束关系, 如图 1 、图 2 所示: 例如对于多电子体系, 教学楼的楼层数等于主量子数, 每一楼层的复式层代表电子亚层, 也 即代表角量子数, 因此楼层数与复式层数的数量关系, 也就是主量子数与角量子数的约束关系, 每 一层复式有若干教室, 教室数目代表磁量子数, 即复式层数与教室数的关系, 也代表着角量子数与 磁量子数的约束关系。 


\begin{tabular}{|c|c|c|}
\hline \multirow{4}{*}{$\begin{array}{c}n \text { 楼 } \\
n\end{array}$} & 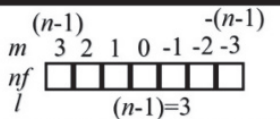 & 复式四层 \\
\hline & $\left.\left.{ }_{n}^{m}{ }_{n}^{2}{ }_{n}^{2}\right|_{2} ^{10}\right|^{-1-2}{ }^{-2}$ & 复式三层 \\
\hline & 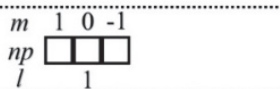 & 复式二层 \\
\hline & $n s \stackrel{m=0}{\square}$ & 复式一层 \\
\hline \multirow{3}{*}{$\begin{array}{c}\text { 三楼 } \\
n=3\end{array}$} & $\left.\left.{ }_{3}^{m} \frac{2}{1}\right|_{2} ^{-10}\right|^{-1-2}$ & 复式三层 \\
\hline & ${ }_{3 p}^{m} \square_{1}^{10} \square^{0}$ & 复式二层 \\
\hline & $\begin{array}{c}m=0 \\
3 s \stackrel{m}{\square}=0\end{array}$ & 复式一层 \\
\hline \multirow{2}{*}{$\begin{array}{c}\text { 二楼 } \\
n=2\end{array}$} & ${ }_{2 p}^{m} \square_{1}^{10} \square^{-1}$ & 复式二层 \\
\hline & 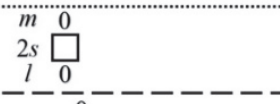 & 复式一层 \\
\hline $\begin{array}{c}\text { 一楼 } \\
n=1\end{array}$ & $1 s \stackrel{m=0}{\square}$ & \\
\hline
\end{tabular}

图2 多电子体系 “虚拟教室与教学楼” 模型

另外, 我们也利用 “虚拟教室与教学楼” 模型向学生讲授电子排布的三大规则: 能量最低原理、 泡利不相容原理和洪特规则, 引导学生掌握1-18号元素的电子排布规律。

在课堂上采用以上类比教学, 大部分学生对四个量子数的概念及其物理意义在头脑中产生了清 晰的图像, 也初步地理解了1-18号元素的电子排布规律, 说明我们类比教学取得理想的成果。但是 单凭以上的知识, 学生还不足以完全掌握其他基态原子的电子排布。对于多电子原子, 原子轨道径 向分布的不同, 导致了屏蔽效应和钻穿效应的出现, 引起了多电子原子能级交错的现象。例如原子 序数为 15-20的元素, $E_{4 s}<E_{3 d}$; 原子序数大于 21 的元素, $E_{3 d}<E_{4 s}$ 。由此可见, 多电子原子的能级次 序是比较复杂的, 导致学生难以判断能级次序, 特别是经常忘记 $4 s$ 与 $3 d$ 轨道的能量大小比较, 更难 以掌握19号元素以后基态原子的电子排布。为了帮助学生解决这一问题, 我们在课堂上使用了如图 3所示的Pauling电子填充能级顺序图向学生讲授19-36号元素的基态原子电子排布规律。

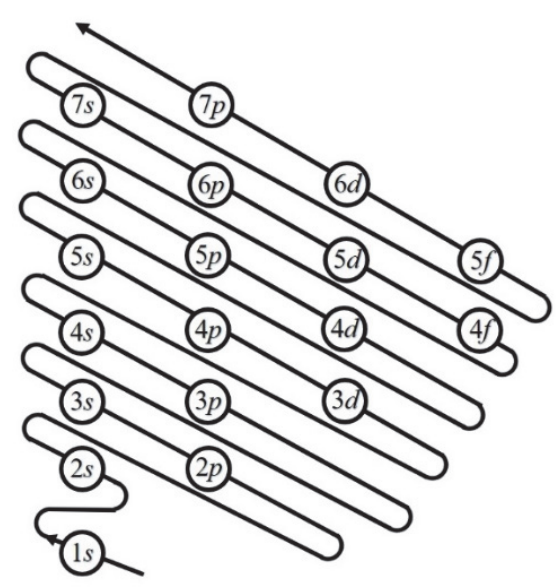

图3 电子填充能级顺序图 
最后, 为了检验本次教学改革的效果, 我们进行了随堂测验, 考查1-36号的元素的基态原子的 电子排布。发现与往年相比, 学生都普遍获得较好的成绩, 说明采用这种类比教学方法取得满意的 效果。说明这种把抽象的概念具象化、形象化的类比教学法取得了成功。同时, 我们在无机化学后 续内容的教学中逐渐加强培养学生的抽象思维。

\section{4 结语}

提出问题: 一直以来, 不少学生反映无机化学课程的物质结构部分难学难懂, 特别是觉得四个 量子数非常抽象而学得一知半解, 导致对后续的内容完全不知所云。

解决问题: 为了帮助学生解决这一难题, 我们采用类比教学的方法将抽象的四个量子数的内容 具象化一一利用 “虚拟教室与教学楼” 模型进行类比, 将电子比作学生, 楼层比作电子层, 楼层中 的复式比作电子亚层, 一间教室比作一个原子轨道, 将看不见摸不着的抽象概念形象化、简单化。 同时, 向学生推荐使用类比法将抽象的内容具体化, 能帮助学生培养抽象思维, 帮助学生适应大学 的学习。

课后反响: 通过学生的课堂表现与随堂测验的结果, 发现学生的理解和掌握程度比往年都要理 想, 说明当前的类比教学取得理想的教学效果。这也激励我们今后将类比教学方法运用于更多的知 识点教学上, 帮助学生理解得更深入, 掌握得更扎实。同时也希望学生能够将类比思维应用于日后 的学习与科研之中。

不足: “虚拟教室与教学楼” 模型也存在其不足之处:

(1) 未能强调自旋并无经典的可对应量;

(2) 未能体现洪特规则;

（3）没法解释屏蔽效应和钻穿效应带来的多电子原子轨道能级交错;

(4) 该模型使用是否为复式楼层去类比单电子与多电子体系, 实际上, 原子或分子体系是不分 单电子或多电子体系, 两者应是统一自洽的, 本模型并没有建立一个更完善的模型去统一描述单电 子与多电子体系, 这是本模型的缺点。但是如果建立这样的模型, 势必需要引入介绍更深奥难懂的 概念, 例如两个电子之间的交换相关作用等, 只会增加学生的理解难度。如果我们讲授的是本科高 年级课程结构化学, 甚至是研究生课程量子化学, 引入更深奥的概念去构建更完善的模型是很有必 要的。但我们的初衷只是为了让刚踏入校园的大一新生掌握四个量子数的物理意义以及其能级的能 量高低, 才使用 “虚拟教室和教学楼” 模型进行类比, 如果引入更深奥的理论去构建更完善的模型, 我们认为这是不切实际的, 学生肯定很难理解和接受。当然, 我们在讲授的过程中会以思考题的形 式让学生寻找该模型的缺点, 最后我们肯定也会在讲授过程中强调其中的缺点, 以防学生进入理解 误区。

这是当前 “虚拟教室与教学楼” 模型的不足, 这也是在后续的教学实践中需要提高与改进之处。 在这里抛砖引玉，希望能跟同行进行交流并改进。

\section{参 考 文 献}

[1] 宋天佑, 程鹏, 王杏乔, 徐家宁. 无机化学(上册). 第2版. 北京: 高等教育出版社, 2009: 121-127.

[2] 唐风, 唐利强. 当代教育理论与实践, 2014, 6(5), 21.

[3] 郭玉鹏. 大学化学, 2011, 26(6), 67.

[4] 杨风霞, 连照勋. 大学化学, 2012, 27 (4), 23.

[5] 黄四平, 于占江, 刘洋, 黄怡, 王晓芳. 大学化学, 2016, 31 (8), 17.

[6] 唐正姣, 李萍, 陈苏芳, 覃远航. 大学化学, 2018, 33 (12), 89. 
[7] 徐光宪, 黎乐民, 王德民. 量子化学基本原理和从头计算法(上册). 第2版. 北京: 科学出版社, 2007: 13-15.

[8] 周公度, 段连运. 结构化学基础. 第4版. 北京: 北京大学出版社, 2008: 25.

[9] 朱洪玉. 大学物理, 1988, 1 (7), 7.

[10] 姚久民, 石凤良. 唐山师范学院学报, 2005, 27 (5), 67.

[11] 粟一匡. 怀化师专学报(自然科学版), 1986, No. Z1, 79.

[12] 黄笃之, 贺锋. 现代物理知识, 1995, 7 (1), 33.

[13] 鲁吉之. 化学教学, 1988, 3, 37 .

[14] 王国栋. 科技资讯, 2008, 12, 237.

[15] 郭保章. 化学教育, 1990, 11 (1), 56. 